\title{
CITIZEN SCIENCE FOR EARTH OBSERVATION: APPLICATIONS IN ENVIRONMENTAL MONITORING AND DISASTER RESPONSE
}

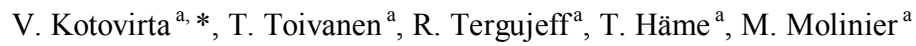 \\ ${ }^{a}$ VTT Technical Research Centre of Finland, P.O. Box 1000, FI-02044 VTT Espoo, Finland, firstname.lastname@vtt.fi
}

\author{
Commission VI, WG VI/4
}

KEY WORDS: citizen science, Earth observation, forest biomass, disaster mapping, water quality

\begin{abstract}
:
Citizen science is a promising way to increase temporal and spatial coverages of in-situ data, and to aid in data processing and analysis. In this paper, we present how citizen science can be used together with Earth observation, and demonstrate its value through three pilot projects focusing on forest biomass analysis, data management in emergencies and water quality monitoring. We also provide recommendations and ideas for follow-up activities.
\end{abstract}

In the forest biomass analysis pilot, in the state of Durango (Mexico), local volunteers make in-situ forest inventory measurements with mobile devices. The collected data is combined with Landsat-8 imagery to derive forest biomass map of the area. The study area includes over 390 permanent sampling plots that will provide reference data for concept validation and verification.

The emergency data management pilot focuses in the Philippines, in the areas affected by the typhoons Haiyan in November 2013 and Hagupit in December 2014. Data collected by emergency workers and citizens are combined with satellite data (Landsat-8, VHR if available) to intensify the disaster recovery activities and the coordination efforts. Simple processes for citizens, nongovernmental organisations and volunteers are developed to find and utilize up to date and freely available satellite imagery for coordination purposes and for building new not-for-profit services in disaster situations.

In the water quality monitoring pilot, citizens around the Baltic Sea area contribute to the algae situation awareness by collecting algae observations using a mobile application. In-situ observations are compared with surface algal bloom products based on the satellite imagery, e.g. Aqua MODIS images with 500 meter resolution. As an outcome, the usability of the citizen observations together with satellite data in the algae monitoring will be evaluated.

\section{INTRODUCTION}

Observations of our environment provide the foundation to understand and tackle global issues like deforestation, ozone hole, air pollution and climate change. Monitoring activities are essential for analysing, modelling and forecasting of the state of our planet. Satellite remote sensing provides means to monitor the planet at the global scale and in a consistent manner. Efficient utilization of satellite based Earth observation benefits from validation and complementary data provided by terrestrial observations from various sources. However, spatially and temporally dense terrestrial observation networks are difficult and costly to implement.

Citizen contributions to environmental monitoring are increasing (Conrad et al., 2011). This is part of a broader emerging field of citizen science in which citizens produce scientifically meaningful observations or analyses (Haklay, 2012). Citizen science has been successfully applied to ecological research, e.g. for monitoring birds, insects and invasive plants (Dickinson et al., 2012) as well as for analysing Google search queries to track influenza (Ginsberg et al., 2012). Citizens have been involved in environmental monitoring, to some extent, for over a hundred years. For example, the Christmas Bird Watch, started by ornithologists of North America, has been ongoing since 1900 (Haklay, 2012).

Recent advances in information and communication technology (ICT) and increased awareness of the status of the environment, in particular global climate change, have activated people even more to participate in monitoring (Burke et al., 2006). Nowadays mobile devices are present wherever there are people, which enables new possibilities in building platforms for utilizing citizen observers as spatially and temporally dense observation networks. This opportunity is strengthened by the growing common concern about the state and future of the environment. People are increasingly willing to contribute their time and resources to the analysis and processing of scientifically collected data and also to collect observations about the environment. Currently, there are studies on how citizen science could be combined with Earth observation, and for example NASA has a dedicated web page for various citizen science projects that utilize citizens in collecting data sets to be used with Earth observation data.

In this paper we present three pilot projects demonstrating utilization of citizen science for Earth observation and provide recommendations and ideas for follow-up activities.

\footnotetext{
* Corresponding author.
} 


\section{PILOT PROJECTS}

\subsection{Forest Biomass Analysis}

The forest biomass analysis pilot project demonstrates a novel concept in which volunteers gather forest inventory data that can be used as in-situ data for forest biomass estimation from satellite imagery. The collected data includes stem biomass, tree species, tree height and age (Molinier et al., 2011).

The pilot project uses a mobile forest measurement tool called Relasphone that is developed by VTT Technical Research Centre of Finland (Molinier et al., 2014). The Relasphone concept has been developed to help forest owners and timber brokers make forest measurements. Screenshots of the application are shown in Figure 1. The biomass estimation is based on the relascope functionality (Bitterlich, 1984). The basal area per hectare of the cross-section of the trees is measured by counting trees that fill a narrow gauge. The counting is done from a fixed location, a sample plot, from a $360^{\circ}$ circle. By combining the basal area information with tree height and applying empirical equations, the tree growing stock volume and biomass can be estimated. The forest measurements are combined with remote sensing data to produce a forest biomass map. Measurements include GPSbased location information and thus provide a cost-efficient reference data source for satellite image interpretation.
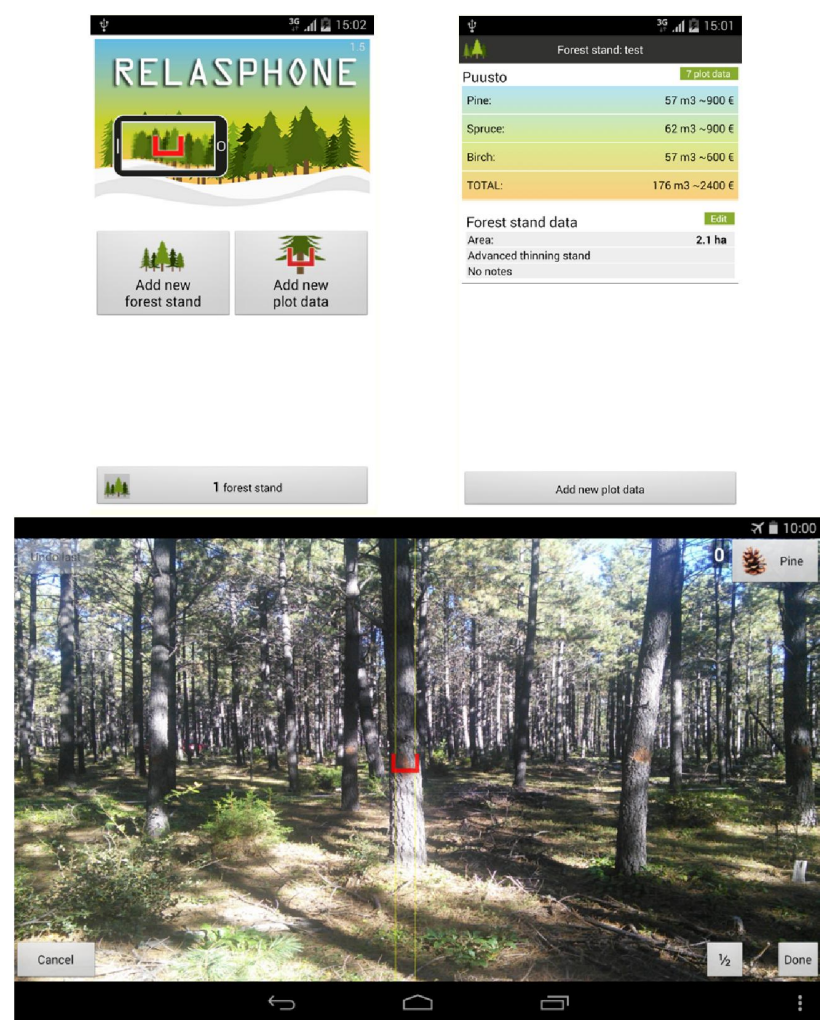

Figure 1: Relasphone application for in-situ forest inventory data collection. Main screen, relascope view, and forest plot summary (biomass and value).

The pilot demonstration is implemented in the Mexican state of Durango, where the study area includes over 390 permanent sampling plots that will provide reference data for concept validation and verification. The local volunteers use the Relasphone application to collect measurements of the forest parameters. During November and December 2014, 55 plots with existing reference measurements were visited, and basal areas were measured at those locations with Relasphone. Data were compared to the reference forest inventory data for accuracy assessment, and preliminary results indicate good agreement between Relasphone measurements and reference forest inventory data. The collected data are combined with Landsat- 8 imagery to derive forest biomass map of the area. Results of the data analysis will be published later in a separate study.

Our initial analysis suggests that citizen science can improve forest in-situ data availability for Earth observation. Citizenscitizens can provide reliable data which can be easily utilized in satellite image processing. However, citizen observations using this method cannot yet fully replace traditional forest inventory, since empirical models, based on measurement of individual trees, are needed to transform the Relasphone observations into biomass. In many countries such equations are already available but in the context of this study they are computed in in Mexico. However, Relasphone can already contribute to filling the gaps in in-situ data availability. Users reported that the tool has "a great data acquisition capacity in forest stands when undergrowth is not too dense to block visibility and a good sensibility to changing conditions within a forest stand", and they think that Relasphone could be "a good working tool from the operational point of view".

The overall vision related to the pilot is to develop the concept of collecting in-situ data for biomass analysis to a widely accepted and recognized method. The pilot concept supports participatory forest management planning and sustainable forest management. The quality and reliability of citizen data should be well known and all the data including quality estimation should be provided to the main systems such as Copernicus land service and GEOSS through standard interfaces.

The concept is expected to be very cost-effective and flexible in comparison to the present forest management planning procedures. If the concept is successful, it could change the way forest databases and management plans are produced and lead to major cost reductions. Improved forest management planning supports also sustainable management and use of forest.

\subsection{Emergency Data Management}

The goal in the emergency data management pilot is to study and verify in various pre- and post-disaster scenarios the usability and usefulness of combining information from three complementary sources: (a) verified information collected by professionals; (b) citizen science based information, including social networks and crowdsourcing; and (c) up-to date satellite data.

In this pilot the target area for Earth observation and citizen science data collection was Eastern Visayas in the Philippines. The recent major natural disasters hit this area the most in the Philippines. Citizen science data were and still are collected after typhoons Haiyan (11/2013) and Hagupit (12/2014) using the Poimapper mobile data collection platform (http://www.poimapper.com/) (Figure 2). 
In the pilot we validate the relevancy and explore the accuracy and efficiency of citizen-borne information in disaster risk reduction and disaster response situations. We will compare the results of traditional approach where data are collected by trained staff of response organizations with the results of data collected by citizens. We expect an increased value through faster, better targeted and highly efficient disaster data management procedures to better support the ground operations in emergency situations.
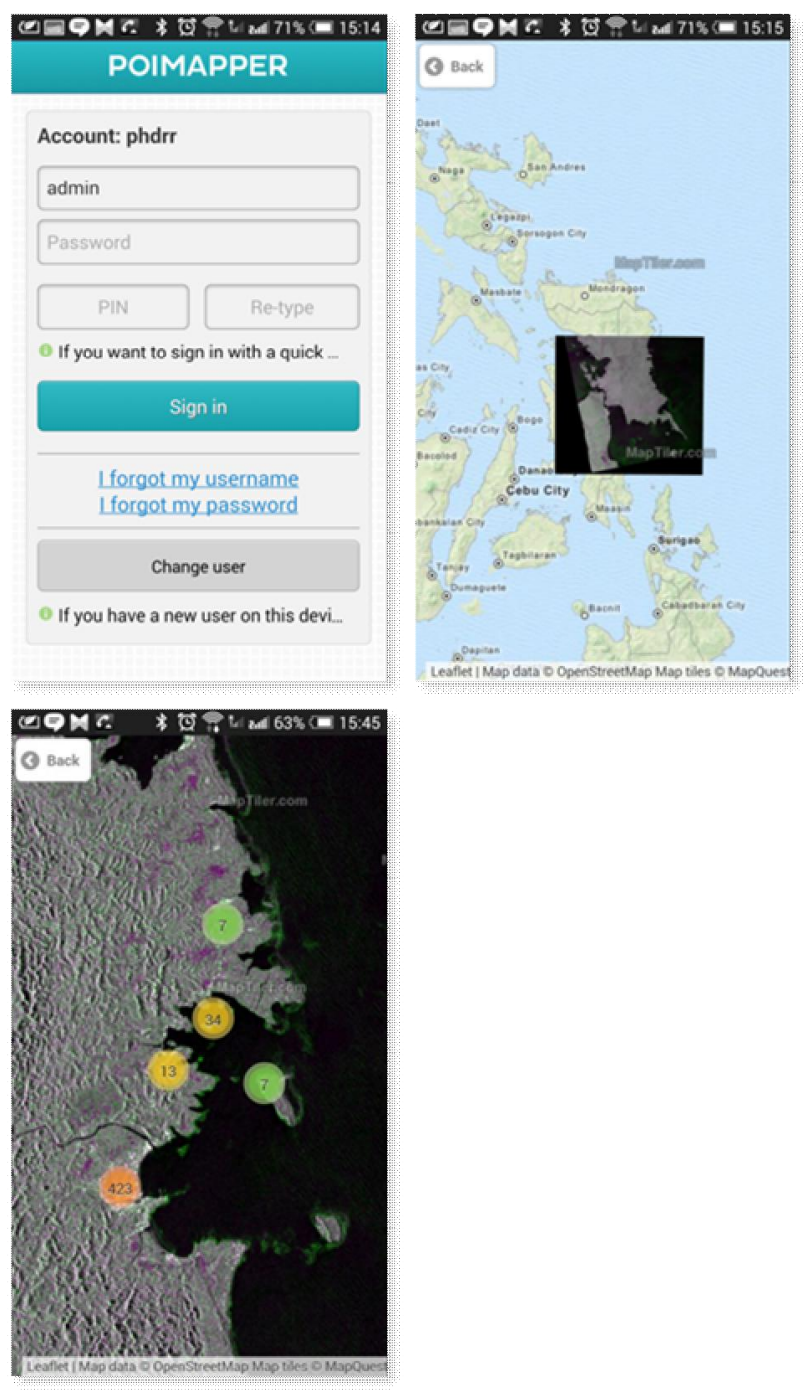

Figure 2: Poimapper application used in the emergency pilot for collecting observations by emergency workers and citizens.

\subsection{Water Quality Monitoring}

In the water quality monitoring pilot, we concentrate on algae monitoring, as the intensity of cyanobacterial blooms is used in the assessment of the ecological status of surface water bodies. Citizen science can complement the information about the algae conditions, especially on the shoreline and in lakes, where the use of satellite remote sensing has limitations and/or phytoplankton monitoring is infrequent or totally lacking.

Citizens have been engaged to make algae observations in the Baltic Sea and Finnish waters since 2011. Two systems are currently in use: a mobile phone application called Levävahti (Algae Watch) and a web-based lake information system called Järviwiki. In order to get citizens involved, the applications have been marketed in various public water-related events and exhibitions, on the web, and via local and national news services at the beginning of the summer algal growth season in the Baltic Sea area. Around 400-800 citizen observations have been collected yearly and the analysis of citizen algae observations in 2011-2013 has been reported (Kotovirta et al., 2014). In 2014, users from various registered associations were trained to use the algae monitoring application to identify and report algae observations. These trained users, in turn, trained more users in their associations. During the summer 2014, 360 mobile citizen algae observations were collected.

In the pilot we are studying how citizen observations can be used in combination with Earth observation data, i.e. to validate or complement satellite-based products. We have started the study by comparing citizen data with the surface algal bloom image products derived from satellite data. For example, the Finnish Environment Institute publishes in the web various data products related to water quality, mainly from Aqua MODIS (500 $\mathrm{m}$ resolution) and MERIS $(300 \mathrm{~m}$ resolution) instruments. In the examples below we used data from the year 2011 when the amount of algae was at the highest level since the citizen observations started. We used two image products with the resolution of 300 meters based on the MERIS satellite instrument.

Figure 3 and Figure 4 present the citizen algae observations mapped on top of the MERIS based surface algal bloom product from the Baltic Sea near Finnish coastline. The red colour shows areas with extremely plenty of algae, yellow shows areas with plenty of algae, turquoise shows areas with some algae, and blue represents areas without algae. Citizen observations which are presented as dots in the figures use the same colour coding. Figure 4 shows how citizen observations can be utilized to complement the situational picture of the overall algae conditions in areas which are out of the reach of satellite-based products. 


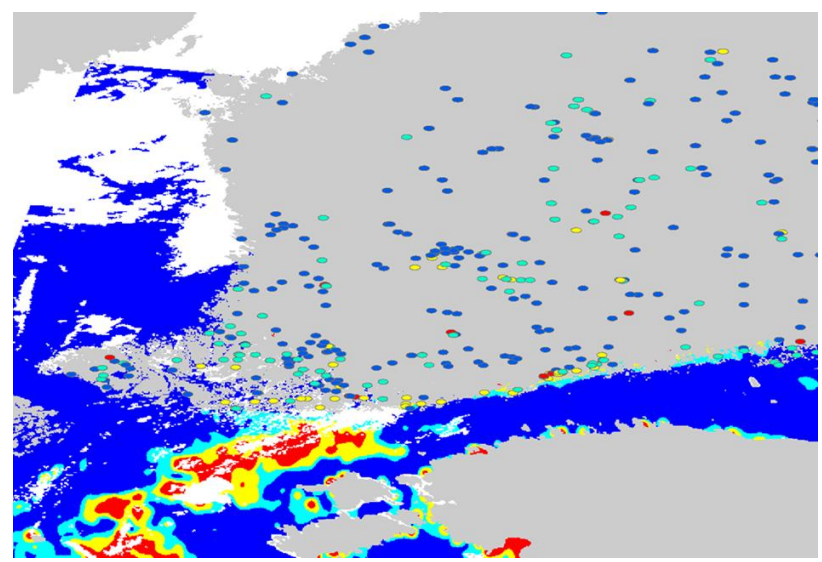

Figure 3: Citizen observations mapped on top the surface algal bloom product by the Finnish Environment Institute from summer 2011 based on MERIS data.

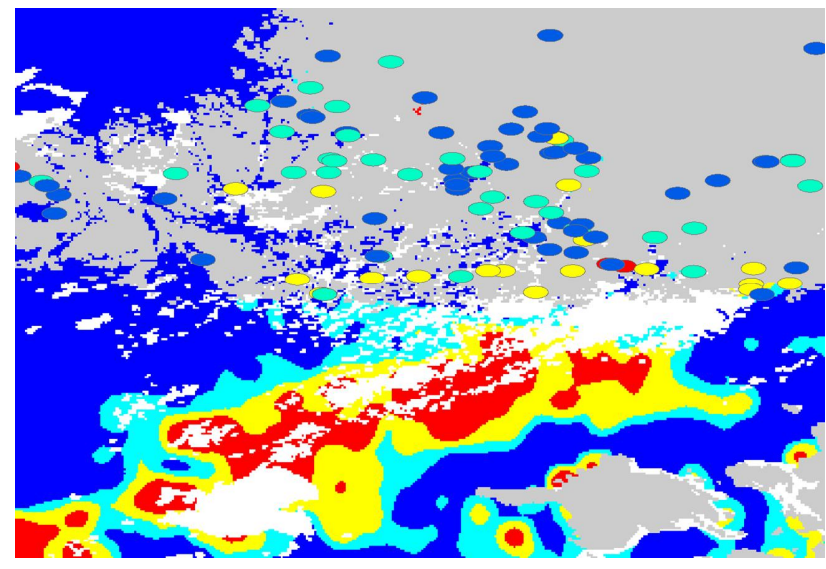

Figure 4: A detailed view of southern part of Finland to demonstrate how citizen observation can complement remote sensing based product (by the Finnish Environment Institute).

The problem with the current satellite image products is that the resolution of the remote sensing instruments is too coarse to enable accurate maps in the Baltic Sea shoreline and the inner lakes of Finland, exactly where the citizens are mainly making the observations. Monitoring of small water bodies by satellite remote sensing requires images with a good spatial resolution $(<30 \mathrm{~m})$ which are currently not operationally available on a daily basis. The remote sensing products will improve when Sentinel-2 and Sentinel-3 data are available, which will enable more accurate mapping of shorelines and inner lakes using remote sensing.

Europe is facing increasing monitoring requirements to meet obligations under, for example, the Water Framework Directive and the UN Convention on Biological Diversity (CBD). This opens up opportunities to develop and test new innovations for citizen science and community-based environmental monitoring. These innovations should not only provide new ways of gathering data but also engage and encourage the community in sustainable management of the environment.

The preliminary results show that citizen observations can be used to increase spatial and temporal coverage of national algae monitoring. More research is needed to analyse reliability of citizen observations more thoroughly.

\section{FUTURE DIRECTIONS, CHALLENGES AND OPPORTUNITIES}

Citizen science in the Earth observation domain is still in an early phase of development and the nature of current projects is typically experimental. Most activities are initiated by the scientific community - universities, research institutes and nature conservation/observation networks. There are only a few examples of citizen science projects that are operated on a well- organized high quality (i.e. professional) level. Outside of the Earth observation domain, successful examples with high level of professionalism include SETI@home and Zooniverse.

New developments are expected to provide tools for more efficient use of citizen science also for Earth observation. The on-going open data trend aims at providing more and more environmental data free-of-charge. These data include also citizen science and Earth observation data, and the availability of new data is likely to boost the development of new applications combining the two data sources. There will be more citizen science data available for Earth observation applications and more satellite data to citizen science projects. Other promising opportunities include e.g. exploiting the growing number of citizen owned/controlled unmanned aerial vehicles (UAVs), application of gamification in the design of citizen science activities, and utilizing external sensors (e.g. air quality, water quality) attached to mobiles phones.

\subsection{Vision 2020}

Based on the current development trends, citizen science in the year 2020 can be expected to be a widely accepted method for collecting good quality in-situ data and for providing a supplementary data source for Earth observation product validation, development and use. Currently, some citizen science activities have established as independent non-profit organizations and a few even as companies. This trend is likely to continue, and towards 2020 we can expect a more widespread launch of professionally organized citizen science activities, aiming at operation as a valid business.

The increased professionalism implies stricter data quality requirements, and better defined customer sectors, citizen user groups and associated value networks. In general, both the operational costs and benefits will be higher, but this setting enables citizen science to function sustainably as a business on its own, producing value for customers in terms of enabling more low-cost production of needed data, and extending the scope of use of the acquired data.

In 2020, newly established citizen science companies provide and maintain technology and services for making measurements, collecting data, ensuring data quality and delivering data to the users, i.e. their customers. They also maintain citizen science communities and take care of dedicated user engagement, by delivering feedback to citizens, providing suitable incentives to ensure motivation, and enabling discussions about campaigns and the environment. New in-situ monitoring innovations are introduced and provide new business opportunities.

In 2020, satellite imagery can be expected to be in broad use in many citizen science activities. Space agencies, such as the 
European Space Agency (ESA), and research organizations provide support for citizen science to facilitate Earth observation data utilization. Organizations managing citizen science activities run monitoring campaigns, effectively motivating citizens to start and continue making observations, and mediate discussions on the needs between data users and citizen observers.

Different organizations participate in the implementation of new citizen science methods: technology developers and service providers, organisations managing citizen science campaigns and motivating the participating citizens, citizens associations with direct and efficient communication channels with the citizens, environmental agencies and research organizations defining environmental monitoring requirements, and organizations involved in political decision making to define requirements and making impact.

The citizen produced data are available via state-of-the-art data sharing platforms such as Copernicus and GEOSS to environmental institutes and researchers for enhanced environmental monitoring and analysis of the environmental conditions. The data are available for improved decision making on many levels in politics, industry and citizen activities, leading to enhanced implementation of governance and global policy objectives. Citizen science will also have an important role in educating and encouraging citizens to participate in scientific research, especially, it will inspire young people towards science.

\subsection{Challenges}

Before the above vision can be realized, some identified challenges of citizen science need to be tackled. Here, we consider two of them that have appeared relevant in the pilot cases: citizen motivation and data quality.

\subsubsection{Motivation}

In order for citizen science activities to remain successful for extended periods of time there should be adequate motivation for the citizens to participate. In many projects, there are no motivation mechanisms except relying on the citizen users' goodwill, or the participation is based on one's hobby or personal ideology. Many projects are simply offering the participants a chance to assist researchers in achieving results, which may not guarantee the sustainability of the activity.

Many approaches for motivation are suggested, for example, a recruitment framework for identifying potential participants for data collections (Reddy et al., 2010a), an incentive mechanism for stimulating participatory sensing applications (Juong-Sik and Hoh, 2010), and micro-payments as an incentive mechanism (Reddy et al., 2010b).

Suitable incentives differ per project and per user group. A number of approaches for enabling user motivation in observation campaigns have been identified earlier (Kotovirta et al. 2012). These should be studied and developed further to understand how to motivate citizen scientists to participate in projects supporting Earth observation. Relevance: Citizen science campaigns should be meaningful to the user and relate to his or her everyday life and personal interests. Recognition: Users should receive feedback about the significance of their contribution. Status: Users can be assigned the status of a recognized observer through continued contribution activity. Reward: Users could receive reward, either monetary or service value, or exclusive information not accessible elsewhere. Social linking: Users should be able to connect and share experiences with peer contributors. Reminders: User activity can be revitalized by reminding the users and by inviting them to new campaigns. Simplicity: The success and deployment of participatory sensor networks depends on price, usability and versatility of the participatory sensing software and devices.

\subsubsection{Data quality}

Some quality issues often limit the usability of citizen data in scientific use: the data quality varies, it is lacking documentation, it fails to follow scientific principles of sampling design, or its coverage is incomplete (Goodchild and $\mathrm{Li}, 2012$ ). When citizen science is applied to remote sensing, the quality and reliability of the data produced by citizens is relevant, especially when the data are used as ground-truth for evaluating, validating, and calibrating models.

The data quality may vary, but when it is estimated and documented in the metadata, the data can be used in the right context in the applications. For example, some citizens may use qualified devices to make high quality measurements suitable for high quality scientific work, whereas some citizens may give only subjective estimations of some parameters with varying quality. Even low quality observations can be useful if the number of observations is high enough for calculating averages and other statistics to be used in the application. There are methods for encapsulating uncertainties in the data, like UncertML (http://www.uncertml.org/).

\section{CONCLUSIONS}

Citizen science is a promising way to increase both temporal and spatial coverages of in-situ data and to process and analyse data in the domain of Earth observation. We have studied the use of citizen science data together with Earth observation data in three different pilots focusing on forest biomass analysis, data management in emergencies and water quality monitoring.

In all the pilot cases, citizen observations bring additional value, which suggests that citizen science can be valuable to Earth observation applications. We estimate that there will be professionally organised citizen science projects, new business emerging around citizen science, and the number of applications that combine citizen science and Earth observation growing. However, we see that two challenges need to be tackled more carefully before citizen science can be in extensive use in Earth observation: citizen motivation for continuous and thorough citizen observation campaigns, and data quality estimation and documentation for more rigorous use of the data in the applications.

\section{ACKNOWLEDGEMENTS}

This work has been conducted in the EducEO project (contract N.: 4000111132/14/I-AM) funded by European Space Agency (ESA), and in the Measurement, Monitoring and Environmental Assessment research programme funded partly 
by Tekes, the Finnish Funding Agency for Technology and Innovation and partly by the CLEEN industry consortium.

\section{REFERENCES}

Bitterlich, W., 1984. The Relascope Idea: Relative Measurements in Forestry, Commonwealth Agricultural Bureaux, Farnham Royal, 1984.

Burke, J., Estrin, D., Hansen, M., Parker, A., Ramanathan, N., Reddy, S., Srivastava, M.B., 2006. Participatory Sensing. In: Proceedings of the International Workshop on World-SensorWeb (WSW'2006), ACM, Boulder, CO, USA, 31 Oct 2006.

Conrad, C., Hilchey, K.. 2011. A review of citizen science and community-based environmental monitoring: issues and opportunities. Environmental Monitoring and Assessment 176: 273-291. doi:10.1007/s10661-010-1582-5

Dickinson, J.L., Shirk, J., Bonter, D., Bonney, R., Crain, R.L., Martin, J., Phillips, T., Purcell, K., 2012. The current state of citizen science as a tool for ecological research and public engagement. Frontiers in Ecology and the Environment 10(6): 291-297

Ginsberg, J., Mohebbi, M.H., Patel, R.S., Brammer, L., Smolinski, M.S., Brilliant, L., 2012. Detecting influenza epidemics using search engine query data Nature, 457, February (2009), pp. 1012-1014.

Goodchild, M. F., Li, L., 2012. Assuring the quality of volunteered geographic information, Spatial Statistics, Volume 1, May 2012, Pages 110-120, ISSN 2211-6753, http://dx.doi.org/10.1016/j.spasta.2012.03.002.

Haklay, M., 2012. Citizen Science and Volunteered Geographic Information - overview and typology of participation. In: Sui DZ, Elwood S, Goodchild MF (eds) Volunteered Geographic Information, Public Participation and Crowdsourced Production of Geographic Knowledge, Springer, Berlin.

Juong-Sik, L., Hoh, B., 2010. Dynamic pricing incentive for participatory sensing. Pervasive and Mobile Computing 6 (6): 693-708.

Kotovirta, V., Toivanen, T., Tergujeff, R., Huttunen , M., 2012. Participatory Sensing in Environmental Monitoring Experiences. Sixth International Conference on Innovative Mobile and Internet Services in Ubiquitous Computing, pp. 155-162.

Kotovirta, V., Toivanen, T., Järvinen, M., Lindholm, M., Kallio, K., 2014. Participatory surface algal bloom monitoring in Finland in 2011-2013. Environmental Systems Research $20143: 24$.

Molinier, M., Andersson, K., Hame, T., 2011. Automatic tree stem delineation supporting forest inventory. Geoscience and Remote Sensing Symposium (IGARSS), 2011 IEEE International, vol., no., pp. 4465-4468, July 2011. doi: 10.1109/IGARSS.2011.6129458

Molinier, M., Häme, T., Toivanen, T., Andersson, K., Mutanen, T., 2014. Relasphone - Mobile phone and interactive applications to collect ground reference biomass data for satellite image analysis. Geoscience and Remote Sensing Symposium (IGARSS), 2014 IEEE International, vol., no., $\begin{array}{lllll}\text { pp.836,839, } 13-18 \quad \text { July } 2014 . & \text { doi: }\end{array}$ 10.1109/IGARSS.2014.6946554

Reddy, S., Estrin, D., Srivastava, M., 2010a. Recruitment framework for participatory sensing data collections. In: Proceedings of the 8th International Conference on Pervasive Computing, Helsinki, Finland, pp 138-155.

Reddy, S., Estrin, D., Hansen, M., Srivastava, M., 2010 b. Examining Micro-Payments for Participatory Sensing Data Collections. In: Proceedings of the 12th ACM International Conference on Ubiquitous Computing (Ubicomp '10). ACM, New York, NY, USA, 33-36. 